\title{
Road to Cancer Cure-So Near and Yet So Far
}

\author{
${ }^{1}$ Ravindra Chaturvedi, ${ }^{2}$ Charu Mohan
}

\begin{abstract}
Cancer has become a leading cause of mortality worldwide, next only to cardiovascular diseases. Patients who develop cancer are known to exhibit several genomic alterations. Conventional therapies for the management of cancer have their own limitations. Immunotherapy is coming up as a promising new option for millions of patients who are suffering from end stage metastasis or refractory malignancies. To this end, the goal of cancer immunotherapy is to recruit and potentiate immune cells that can generate a robust and long lasting immune response and specifically target the cancer cells with minimum damage to normal cells. These treatment strategies, when used either alone or in combination, are known to significantly effect tumor growth and have even produced cures. In this brief review, some of the most popular cancer immunotherapy approaches shall be reviewed. Recent data from clinical trials suggest that combining more than one immunotherapy mechanisms, in conjunction with other treatment options like chemotherapy, radiotherapy and targeted therapy, may be the way forward to a complete cancer cure.
\end{abstract}

Keywords: Cancer, CAR- T cell, Checkpoint Inhibitors, Immunotherapy, Monoclonal antibodies.

How to cite this article: Chaturvedi R, Mohan C. Road to Cancer Cure-So Near and Yet So Far. J Med Acad, 2018;1(2): 94-101.

\section{Source of support: Nil}

Conflict of interest: None

Cancer is a complex illness that has become a leading cause of death worldwide, next only to cardiovascular diseases. The latest global statistics estimate the burden of cancer to rise beyond 18 million new cases and 9.8 million deaths annually by the end of 2018. Cancers have diverse genetic, environmental and lifestyle factors contributing to its etiology. Patients who develop cancer exhibit several genomic alterations which work along with epigenetic forces to drive tumor evolution and metastasis. ${ }^{1,2}$ With

\footnotetext{
${ }^{1}$ Dean and Professor, ${ }^{2}$ Associate Professor

${ }^{1}$ Department of Anesthesiology, Army College of Medical Sciences, New Delhi, India

${ }^{2}$ Department of General Medicine, Army College of Medical Sciences, New Delhi, India
}

Corresponding Author: Charu Mohan, Associate Professor, Department of General Medicine, Army College of Medical Sciences, Delhi Cantonment, New Delhi, India, Phone: +919810946939, email: charum88@gmail.com the advancements in the field of cancer biology, it has now become possible to understand the molecular nature of cancers and wield the genomic information to improve outcomes in the management of this deadly disease.

Traditionally, the management of cancer has rested on three pillars of surgery, chemotherapy and radiation therapy as the standard of care. However, given the heterogeneous nature of tumor cells, these therapeutic modalities have their limitations. Besides, the chemotherapeutic agents which target the rapidly dividing cancer cells, often damage other normal cells of the body, especially the cells of bone marrow and gastrointestinal tract. ${ }^{3}$ One of the major difficulties encountered in overcoming cancer is the fact that cancer cells are derived from our normal cells and share the same DNA and major metabolic pathways. Hence, it poses a unique challenge to design therapies that can specifically target only the cancer cells and spare healthy tissues.

Cancer develops as a result of the accumulation of somatic mutations in the cells. This leads to unregulated growth and expansion of these cells while ignoring the normal signals of apoptosis. Our immune system is naturally equipped to protect our body by the recognition and elimination of any such abnormal cell. Yet cancer develops, despite the best of immunosurveillance mechanisms. This has been attributed to the process of immune-evasion, whereby the cancer cells manipulate various molecular mechanisms to evade the sentinel army of the immune system. For example, certain tumors may downregulate their surface expression of antigens for recognition by the major histocompatibility complex (MHC) molecules, or there may be a heterogeneous expression of multiple antigens by the same tumor. Certain tumors may also 'hide' themselves from killer T cells by inducing anergy through the process of 'tumor tolerance'. In addition, many cancers also directly suppress the various components of the immune response by blocking numerous signaling pathways, leading to an overall impairment in the immune defenses of the body. ${ }^{3-6}$

Since the last few years, efforts were ongoing to revive this impaired intrinsic ability of immune system to fight the cancerous invaders. If the suppressive effect of the cancers could be turned off, and the immune system made more robust, it would be possible to defeat cancer without causing damage to the normal cells. Recent advancements in the field of genomics and gene sequenc- 
ing have made it possible to empower the cells of the immune system with miraculous results being achieved. This has led to the emergence of the fourth pillar in cancer management- "Immunotherapy".

Cancer immunotherapy involves modulating the patient's own immune system to fight against the cancer cells. Presently, cancer immunotherapy is based on two major approaches. The first one is a 'passive approach' based on treatments that enhance the body's immune response and make it stronger. It consists of using cells or antibodies which compensate for any deficient immune functions. These can be made exogenously in the laboratory and transfused to the patients. These therapies include the adoptive transfer of $\mathrm{T}$ cells, tumor-specific monoclonal antibodies, and cytokines. The second approach is an 'active approach' where the host's immune system is challenged with an antigen to generate an antitumor immune response. Hence, the patient's immune system should be able to respond upon being challenged. Another way of stimulating the immune response is to use drugs which inhibit the immunosuppressive actions of the tumors. This would be analogous to removing the 'brakes' applied on the normal immune responses by the tumors. The active therapies include 'vaccination' with tumor antigens, the use of 'dendritic cells' as vehicles for tumor antigen delivery, and 'antibodies' targeting crucial 'checkpoints' of T-cell activation. ${ }^{3,6-8}$ Currently, more than 2,000 clinical trials are ongoing, carrying out research on the various approaches to cancer immunotherapy. They are listed on ClinicalTrials.gov. ${ }^{9}$ Based on the above considerations, some of the promising immunotherapy options are:
- Adoptive cellular therapies (ACT): Tumor infiltrating lymphocytes (TIL), T-cell receptor (TCR)-T cells and chimeric antigen receptor-T cells (CAR- T)

- Checkpoint inhibitors

- Cancer vaccines

- Oncolytic virus

- Therapeutic monoclonal antibodies

- Cytokine-based immunotherapy

Figure 1 depicts the various approaches that are used as a part of immunotherapy to target cancer cells.

\section{Adoptive Cell Therapies}

Adoptive cellular therapy (ACT) is coming up as the most exciting treatment in anticancer immunotherapy. It is a very personalized therapy that involves isolating $\mathrm{T}$ cells from the patient which are then expanded in vitro and subsequently reinfused. The initial demonstration that T-Lymphocyte cells had the potential to treat cancer, came from the success of 'allogeneic stem cell' transplantations. But it was observed that the efficacy of natural $\mathrm{T}$ cells was limited, as the transfused $\mathrm{T}$ cells were polyclonal and lacked either sufficient specificity or adequate numbers to control the tumor. The drawback was obviated by the use of genetic engineering to manipulate the $\mathrm{T}$ cells into expressing unique receptors which would be highly specific. It was a major breakthrough, as now the $\mathrm{T}$ cells could recognize and eliminate tumor cells with high specificity when transfused into the body. In addition, mechanisms for the expansion of existing populations of $\mathrm{T}$ cells were also developed, leading to their increased production in relevant doses. ${ }^{7,8,10,11}$

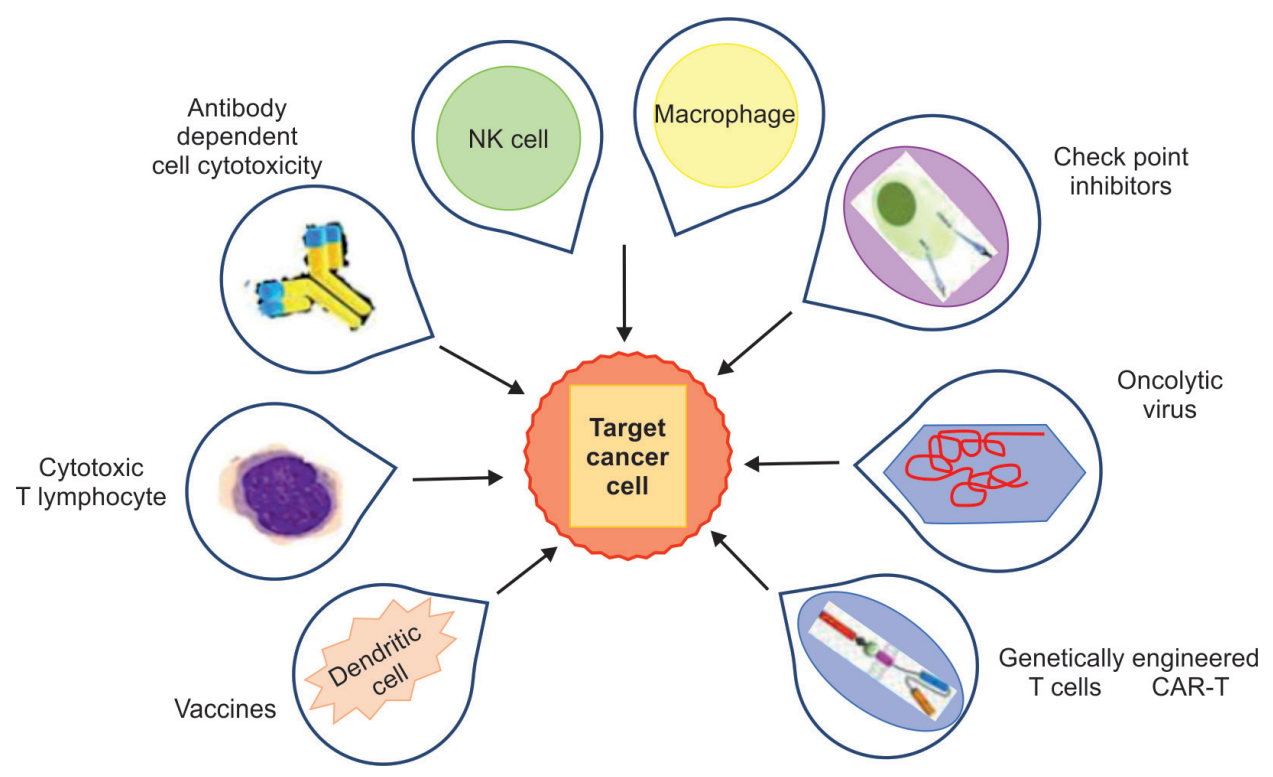

Fig. 1: Strategies used in destroying cancer cells 


\section{Tumor-infiltrating Lymphocytes}

Tumor-infiltrating lymphocytes (TIL) were among the earliest used adoptive therapies. They consist of a heterogeneous population of cells found infiltrated in or around the tumor and include T-cells, B-cells and NK-cells, with the main component of $\mathrm{T}$ cells. A fraction of these $\mathrm{T}$ cells express receptors (TCR) directed against specific tumor antigens, which particularly recognize and kill the tumor cells. However, these 'tumor-specific' T cells are limited in numbers and unable to overcome the cancerous cells. In TIL therapy, these very $\mathrm{T}$ cells are harvested from the resected tumor and then cultured, expanded and overactivated by IL-2 before being reinjected into the patient. The advantage of this therapy is that these specific $\mathrm{T}$ cells are now vast in numbers and all highly trained and supercharged for maximum effect on that tumor. ${ }^{11-13}$

TILs have been used successfully for the treatment of metastatic melanoma. The trials done so far have reported objective response (OR) rates of $38-55 \%$ as evaluated by response evaluation criteria in solid tumors (RECIST), with $22 \%$ patients achieving complete tumor regression (CR). It is also encouraging to know that of these patients who achieved a CR, 98\% have not experienced any recurrences at follow-up times of 5-10 years and are probably cured. It thus appears to be an effective and possibly curative treatment for many patients with metastatic melanoma who can receive it. Although this strategy appears promising there are several limitations to this approach. Firstly, the TIL's are extremely limited in numbers and generating sufficient TIL cells can be a complicated and time-consuming process. Besides, TIL therapy makes it necessary for each patient to have a surgical resection. Also, for reasons that are not fully understood, adoptive transfer of TIL has not shown the desired results in other malignancies. ${ }^{13-15}$

\section{T-cell Receptor-T Cells}

TCR-T cells are $\mathrm{T}$ lymphocytes which are derived from peripheral blood and have been genetically modified and cloned to produce a TCR with targeted affinity. This is achieved by using a retrovirus that encodes a TCR. Here only the extracellular domain of the receptor is modified to provide for a better specificity to the tumor antigen. This confers the lymphocytes, the ability to specifically recognize tumor cells and kill them. These $\mathrm{T}$ cells recognize processed peptide antigens expressed in the context of MHC. However, its utility also becomes limited for this very reason. Since it is MHC restricted for antigen detection and most tumors are known to downregulate the MHC expression, this allows tumor cells to finally escape recognition. Yet, certain advantages of these cells are that they can be produced from peripheral blood T cells (unlike TILs) and can recognize intracellular antigens (unlike CAR-T cells). They have been used with limited success in certain cancers like metastatic melanoma, multiple myeloma, synovial sarcoma, and colorectal carcinoma. ${ }^{15-17}$

In a study by Morgan et al., on patients with metastatic melanoma, a response rate of $13 \%$ was demonstrated (2 out of 15 patients achieved CR). Though this was lower than that achieved by the infusion of autologous TILs $(50 \%)$, the TCR-T- cell therapy has the potential for use in patients for whom TILs are not available. ${ }^{18}$ Another study by Rappaport et al., in patients of multiple myeloma, using the NY-ESO-1-specific TCR-engineered T cells has generated encouraging clinical responses in 16 of 20 patients $(80 \%)$ with advanced disease and a median progression-free survival of 19.1 months. $^{19}$

However, a major concern with this therapy has been the 'on-target/off-tumor' toxicity and decrease in TCR transgene expression over time leading to the ineffectiveness of the therapy. Hence, while higher avidity TCRs may be associated with better tumor responses, the probability for off-tumor toxicities may also be increased. Further clinical studies with probably human-derived affinity-enhanced TCR may be the way forward to overcome the shortcomings. ${ }^{16-18}$

\section{Chimeric Antigen Receptor T cell}

Among the adoptive cellular therapies, the "CAR-T" cells appear to hold excellent prospects for the future. To overcome the limitations of TCR- T cells, 'CAR' technology had been developed. CAR-T cells express an artificial receptor, the chimeric antigen receptor (CAR) on their surface. It is a transmembrane structure with intra- and extracellular parts. It composed of $\mathrm{T}$ cell receptor 'constant domain' (TcR) fused to a B cell antibody's 'variable domain' (V). Hence it confers the T cells with an antibody-like specificity and simultaneously activates them to execute their effector functions. The advantage of such CAR-T cells is that they can recognize full antigens independently from $\mathrm{MHC}$ restriction in contrast to TCR- T cells that are MHC restricted. When targeted to a specific surface tumor antigen, CAR- T cells proliferate and destroy the tumor cells upon antigen contact. $13,16,17,20,21$

In clinical practice, the $\mathrm{T}$ cells are derived from the circulating $\mathrm{T}$ cells in the patient's blood. Subsequently, the patients' tumor is sequenced to determine the best extracellular antibody arrangement and the construct is built. The $\mathrm{T}$ cells are then genetically modified (transduced) by the introduction of TCR genes via viral vectors or CRISPR technology (clustered regularly interspaced palindromic repeats), activated and amplified before 
being reinjected into the patient. This means that each CAR-T cell is very specific since the construction is made to target the patient's particular tumor with high efficacy. This innovation has made substantial progress in the treatment of hematological malignancies. CAR-T cells with specificity for $\mathrm{CD}^{19}$ (anti-CD ${ }^{19} \mathrm{CAR}-\mathrm{T}$ cells) have generated complete and durable remissions in patients with refractory and relapsed B cell malignancies. Specifically, B cell acute lymphoblastic leukemia (B-ALL) has shown the best prospects to date demonstrating response rates up to $90 \%$ and morphologic CR rates of $67-90 \%$ in some of the major trials done so far. ${ }^{16,20,21}$ Whether this therapy would improve overall survival will only be established once long-term data become available.

Although CAR-T cell therapy has shown impressive clinical benefits in hematological malignancies, they have not shown the desired results in solid cancers. A major reason for this has been the limited ability of CAR-T cells to infiltrate and persist in the solid tumor microenvironment and their dose-limiting toxicities. Nonetheless, few studies done on patients with high-risk neuroblastoma reported complete remission in three of 11 patients ( $27 \% \mathrm{CR})$, and in another phase I clinical trial, a CR of $50 \%$ was achieved. ${ }^{22,23}$ In addition, few recent clinical trials have also demonstrated the feasibility and some efficacy of using this therapy for glioblastoma multiforme, which is one of the most aggressive forms of a primary brain tumor. ${ }^{16,24}$

The successful clinical outcomes using CAR-T therapy, however, are not without dangers and may be associated with life-threatening toxicities. A major concern is the cytokine release syndrome (CRS), which occurs due to rapid T-cell proliferation. Other feared complications are neurotoxicity and the 'On-target, off-tumor' toxicity which need further addressal. Therefore, to enhance safety and limit toxicity, special genes, the 'suicide genes' are being introduced in $\mathrm{T}$ cells which can be activated in case of adverse events and also eliminate transduced $\mathrm{T}$ cells after eradication of the disease.

So far three generations of CAR-T cells have been developed which have been given FDA approval and the fourth generation, so-called 'TRUCKs' or 'armored CARs' are undergoing clinical trials. The third generation of these cells exhibits improved durability as well as proliferating capacities. They have variations in the intracellular side with different co-stimulatory molecules attached to it, such as $\mathrm{CD}^{28}$, OX40 and/or 4-1BB to mobilize multiple molecular pathways for efficient $\mathrm{T}$ cell effector functions. Besides this, another notable feature of CAR T cells is their ability to self-amplify and persist in treated patients for long periods of time. They are hence aptly named the 'living biological drugs'. ${ }^{\prime 3,16,17,20,25}$

\section{Checkpoint Inhibitors}

An important function of the immune system is to distinguish self from non-self antigens. Under normal conditions, to prevent autoimmunity, 'checks' or restraint has to be applied at certain points in the course of the immune response, to protect the host from unopposed T-cell activation. This is immune tolerance and the checkpoints are akin to brakes in the immune system. These checkpoint pathways are one of the important mechanisms which the cancer cells use to their advantage to slow down the immune system and prevent it from attacking them.

The molecular receptors, which are crucial in mediating these pathways are cytotoxic T-lymphocyte-associated antigen 4 (CTLA-4) and programmed death protein 1 (PD-1). These are present on a wide range of cell types like Regulator T cells (T-reg), CD4, CD8, B cells and Dendritic cells (DCs) among others. When the ligands (B7-1/2 and PD-L1/L2, respectively), bind to these receptors, 'inhibitory signals' for T cells are generated which decrease the T cell proliferation and cytotoxicity, and increase T cell apoptosis. In cancer patients, the tumor cells upregulate these pathways by producing such ligands which bind the receptors. This activates the checkpoints and keeps the brakes 'on', on the T cells. Both CTLA- 4 and PD- 1 act independently as brakes and operate at different stages of an immune response. Hence the development of drugs which could restore the immune functions by removing the brakes on them was under much focus in clinical trials. ${ }^{16,26-28}$

Specific monoclonal antibodies have been developed which can interrupt the receptor-ligand interactions and revive the $\mathrm{T}$ cell capabilities of the immune system. These antibodies are known as checkpoint inhibitors. Interestingly, ongoing clinical trials have reported that simultaneous blockage of CTLA-4 and PD-1 pathways significantly enhances the anti-tumor reactivity of $\mathrm{T}$ cells. Besides, an advantage of checkpoint inhibitors is the feasibility of using these monoclonal antibodies as off-the-shelf therapy in a spectrum of malignancies. Presently, several drugs are under development; however, only a few have been given FDA approval for certain cancers. The anti-CTLA-4 drug Ipilimumab and anti-PD-1 drugs, pembrolizumab and nivolumab have provided durable and long-term survival benefits in patients with melanoma and non-small cell lung cancer (NSCLC). In addition, nivolumab has been approved for kidney cancer, bladder cancer, head and neck cancer, and Hodgkin's lymphoma. Besides these, the anti-PD-L1, drugs, atezolizumab, avelumab, and durvalumab have been prescribed for treating bladder cancer, Merkel-cell carcinoma, and urothelial carcinomas, respectively. ${ }^{26-30}$

Although checkpoint blockade overcomes many immune-suppressive mechanisms, most patients do 
not benefit from this therapy clinically. Besides, severe immune-related adverse events including pneumonitis, pancreatitis, and colitis have emerged as potential limiting factors. These are relatively infrequent but can limit therapeutic options for some patients. Also, the role of other checkpoints like TIM3 (T cell immunoglobulin and mucin domain-3), LAG-3 (lymphocyte-activation gene-3), B7-1 and TIGIT(also called T cell immunoreceptor with Ig and ITIM domains)also need to be taken into consideration in the process of immune escape of tumors. To overcome the above limitations, complementary immune agonist therapies may be required to be used in the future. ${ }^{10,13,30-32}$

\section{Cancer Vaccines}

Vaccines have traditionally been used as a preventive form of therapy. However, the vaccines available for management of cancer are available in two forms, as preventive and therapeutic vaccines. The preventive vaccines are given to prevent certain viral infections which may otherwise lead to cancer development in the future in genetically predisposed patients. At present, the vaccines that have been approved by the US FDA for prophylactic use are available against human papilloma virus (HPV) which is known to cause cervical cancer and vaccine against hepatitis B virus (HBV) which is known to cause liver cancer.

The 'therapeutic' vaccines, on the other hand, are intended to treat an existing tumor. Hence they are given to patients only after the development of cancer. These vaccines induce tumor-specific or tumor-reactive immune responses in vivo by mobilizing the immune effector mechanisms to specifically attack and destroy only cancer cells and spare normal cells. In essence, therapeutic cancer vaccines are intended to inhibit further growth of advanced cancers and/or relapsed tumors that are refractory to traditional therapies. Conventionally, therapeutic vaccines have been divided into four main categories: (1) cell-based vaccines, (2) peptide vaccines (3) viral vector vaccines and (4) nucleic acid vaccines. The most promising of these till date is the 'cell-based' vaccines among which the dendritic cell (DC) based vaccines have become very popular since they are comparatively safe for use in humans. ${ }^{10,33-35}$

\section{Dendritic Cell Vaccines}

Dendritic cells (DC)are antigen presenting cells which harbor the intrinsic mechanisms to stimulate both adaptive and innate anti-tumor immunity. As such, DCs have been used as the progenitor of cancer vaccines. Two approaches are used currently to design such vaccines 'in vivo' and 'ex vivo'. The first one involves, direct targeting of antigens to DC receptors in vivo and the other one involves ex vivo generation of antigen-loaded DCs. ${ }^{36-39}$
Sipuleucel-T (Provenge) developed by Dendreon Corporation is the first FDA approved autologous DC vaccine that is being used in the treatment of prostate cancer since 2010. It is generated ex vivo. It was approved for use in asymptomatic or minimally symptomatic metastatic hormone-refractory prostate cancer. It was developed by modifying autologous DC to express a 'fusion protein' comprising of prostatic acid phosphatase (PAP) antigen and granulocyte-macrophage colony-stimulating factor (GM-CSF). The PAP antigen is almost universally expressed in prostate cancer cells, and the GM-CSF provides a maturation factor for the DC. ${ }^{40,41}$

The treatment protocol involves extracting the patient monocytes and subjecting them to leukapheresis method to extract the antigen-presenting 'dendritic cell'. These cells are then sent to a laboratory where they are coincubated with the fusion protein, which is taken up, processed, and presented on the cell surface. In this activated antigen-presenting state, the DCs are then sent back to the treating center where they are re-administered to the patient. Three courses of treatment have to be administered over 6 weeks to trigger an immune response. $37,40,41$

Presently, cancer vaccines are still in their infancy and need to go a long way to achieve favorable therapeutic outcomes in various cancers. Hence, identification of immunogenic neoantigens and development of carefully designed immunotherapies which could foil the escape mechanisms of tumors is of paramount importance for the advancement in cancer vaccines.

\section{Oncolytic Virus}

Oncolytic viruses are viruses that can directly "lyse" (destroy) cancer cells and can also activate other cells of the immune system or induce novel non-self-antigen responses by immune cells to eradicate cancer from the body. The advantage of oncoviral immunotherapy is attributed to the specificity of oncoviruses to target tumor cells exclusively and no other replicating cells. They are injected directly into the tumors. Hence they have markedly lower rates of serious adverse effects. In addition, these viruses are less dependent on specific receptor expression patterns and therefore are less susceptible to the resultant mutational or transcriptional resistance that may otherwise occur. Most of the available oncolytic viruses presently are genetically modified to enhance tumor tropism to reduce the virulence for nontumor host cells. The best studied so far are herpes viruses as they are found to possess some native tumor cell tropism too. Hence, the oncolytic virus is an impressive new multifaceted approach, specifically and effectively targeting and destroying malignant cells. However, the only barrier 
to these therapies presently is that they have a limited therapeutic response to currently available delivery techniques. At present, one oncolytic virus, talimogene laherparepvec, or T-VEC (Imlygic), is approved by the FDA for the treatment of metastatic melanoma. ${ }^{8,10,42}$

\section{Monoclonal Antibodies}

Monoclonal antibodies (mAb) were among the first treatments tested and validated as anticancer therapies apart from classical treatments. They target and bind to certain antigens (tumor-specific antigens or tumor-associated antigens) on the surface of cancer cells, leading directly or indirectly to cell death. This can take place by three main mechanisms, (a) direct cell toxicity by blocking downstream signaling pathways needed for tumor cell growth (b) immune-mediated tumor cell killing, e.g., by antibodydependent-cellular-cytotoxicity (ADCC) or a complementmediated-cytotoxicity and c) by blocking angiogenesis. ${ }^{43}$

Rituximab was the first $\mathrm{mAb}$ approved for clinical use in 1997 for lymphoproliferative disorders. Today antibody treatments are one of the most successful strategies for treating patients with hematological malignancies and solid tumors. They have been rightly described as "magical bullets" due to their tumor antigen specificity. They are divided into two types, naked antibodies (not linked to any active factor) and Conjugate antibodies (conjugated with an active factor like a drug, toxin or radioisotope). Conjugated antibodies can be more powerful, but they can also cause more side effects. Despite their clinical efficacy, the therapeutic MAbs are rarely potent enough by themselves and thus are usually administered in combination with chemotherapy. However, their success in causing regression of malignant disease in certain patients with incurable cancers have made them an important part of the therapeutic repertoire in treating various malignancies. ${ }^{43,44}$

\section{Cytokine Therapy}

Cytokines are considered to be major regulators of the immune system and were also used as one of the earliest modalities in the treatment of cancer patients. They are known to stimulate a general immune response as opposed to generating a targeted response to a specific antigen. Presently, only two cytokines, interferon alpha (IFNa) and interleukin-2 (IL-2) have received FDA approval for treatment in cancers. ${ }^{45,46}$

IFNa was the first cytokine approved for the treatment of hairy cell leukemia (HCL) in 1986. Since then it has also been approved as an adjuvant for treatment of high-risk melanoma patients and other refractory malignancies. It works by activating the NK cells and inducing tumor apoptosis. The IL-2, also known as T cell growth factor, acts as an adjuvant too, to facilitate activation of immune cells in the host to finally kill the cancer cells. It does not by itself act on cancer cells.

The cytokines are, however, often associated with severe dose-limiting toxicities. Hence, their clinical application presently is more focussed on combining them with other biological agents to increase their therapeutic efficacy. Also, the search for novel agents that can circumvent these toxicities has led to the development of engineered cytokine mutants, the 'superkines', and the chimeric antibody-cytokine fusion proteins 'Immunokines', which are expected to provide targeted delivery and improved pharmacokinetics in the future management of malignancies. ${ }^{46}$

\section{CONCLUSION}

The interplay between the immune system and cancer is an intriguing one and uncovering diverse approaches to improve cancer immunotherapeutics is an ongoing phenomenon. Great strides have been made at the forefront of cancer management in the past decade, yet it continues to be a major health concern. Challenges have to be met yet, for certain cancers, especially for metastasized and cancers refractory to traditional treatments. Here, Immunotherapy has provided hope to millions of patients and can be considered a revolution in itself.

The future of immunotherapy is in combining various approaches to accomplish the goal of defeating cancer. Simply put, this goal can be accomplished by firstly breaking down the defenses of these cancers and secondly by creating an army of charged immune cells and unleashing them against cancer. In our search for the road that leads to a perfect cancer cure, many paths have been treaded. But to say that we have arrived is short of truth for though the road may seem near, it is yet so far.

\section{REFERENCES}

1. Bray F, Ferlay J, Soerjomataram I, Siegel RL, Torre LA, Jemal A. Global cancer statistics 2018: GLOBOCAN estimates of incidence and mortality worldwide for 36 cancers in 185 countries. CA: a cancer journal for clinicians. 2018 Nov;68(6):394-424.

2. Zugazagoitia J, Guedes C, Ponce S, Ferrer I, Molina-Pinelo S, Paz-Ares L. Current challenges in cancer treatment. Clinical therapeutics. 2016;38(7):1551-1566.

3. Kokate R. A systematic overview of cancer immunotherapy: an emerging therapy; I Pharm Pharmacol Int J. 2017;5(2): 31-35.

4. Vinay DS, Ryan EP, Pawelec G, Talib WH, Stagg J, Elkord E, et al. Immune evasion in cancer: Mechanistic basis and therapeutic strategies. InSeminars in cancer biology 2015 Dec 1 (Vol. 35, pp. S185-S198).

5. Igney FH, Krammer PH. Immune escape of tumors: apoptosis resistance and tumor counterattack. J Leukoc Biol. 2002 Jun;71(6):907-920. 
6. Iranzo J, Martincorena I, Koonin EV. Cancer-mutation network and the number and specificity of driver mutations. Proceedings of the National Academy of Sciences. 2018;115(26):E6010-E6019.

7. Ventola C L . Cancer Immunotherapy, Part 1: Current Strategies and Agents . P T. 2017 Jun; 42(6):375-383.

8. Ming L, Fukun G. Recent updates on cancer immunotherapy. Precision Clinical Medicine, 2018;1(2):65-74.

9. Clinical Trials.gov ; Cancer Immunotherapy Trials. [Cited in Jan 2019]. Available from: https://clinicaltrials.gov/ct2/res ults?term $=$ cancer+immunotherapy and Search $=$ Search.

10. Papaioannou NE, Beniata OV, Vitsos P, Tsitsilonis O, Samara P. Harnessing the immune system to improve cancer therapy. Annals of translational medicine. 2016 Jul;4(14).

11. Rosenberg SA, Restifo NP. Adoptive cell transfer as personalized immunotherapy for human cancer. Science. 2015;348(6230):62-68.

12. Restifo NP, Dudley NE, Steven A. Rosenberg. Adoptive immunotherapy for cancer: harnessing the $\mathrm{T}$ cell response. Nature Reviews Immunology. 2012 Mar 22;12(4):269-281 .

13. Houot R, Schultz LM, Marabelle A, Kohrt H. T-cell-based immunotherapy: adoptive cell transfer and checkpoint inhibition. Cancer immunology research. 2015 Oct 1;3(10):11151122.

14. Besser MJ, Shapira-Frommer R, Itzhaki O, Treves AJ, Zippel DB, Levy D, et al. Adoptive transfer of tumor-infiltrating lymphocytes in patients with metastatic melanoma: intent-totreat analysis and efficacy after failure to prior immunotherapies. Clinical Cancer Research. 2013 Sep 1;19(17):4792-800.

15. Radvanyi LG, Bernatchez C, Zhang M, Fox PS, Miller P, Chacon J, et al. Specific lymphocyte subsets predict response to adoptive cell therapy using expanded autologous tumorinfiltrating lymphocytes in metastatic melanoma patients. Clinical Cancer Research. 2012;18(24):6758-6770.

16. Fesnak AD, June $\mathrm{CH}$, Levine BL. Engineered T cells: the promise and challenges of cancer immunotherapy. Nature Reviews Cancer. 2016 Sep;16(9):566-581.

17. Ping Y, Liu C, Zhang Y. T-cell receptor-engineered T cells for cancer treatment: current status and future directions. Protein Cell. 2018 Mar;9(3):254-266.

18. Morgan RA, Dudley ME, Wunderlich JR, Hughes MS, Yang JC, Sherry RM, et al. Cancer regression in patients after transfer of genetically engineered lymphocytes. Science. 2006 Oct 6;314(5796):126-129.

19. Rapoport AP, Stadtmauer EA, Binder-Scholl GK, Goloubeva O, Vogl DT, Lacey SF, et al. NY-ESO-1-specific TCR-engineered $\mathrm{T}$ cells mediate sustained antigen-specific antitumor effects in myeloma. Nature medicine. 2015 Aug;21(8):914-921.

20. Hartmann J, Schüßler Lenz M, Bondanza A, Buchholz CJ. Clinical development of CAR T cells-challenges and opportunities in translating innovative treatment concepts. EMBO molecular medicine. 2017 Sep 1;9(9):1183-1197.

21. Maude SL, Frey N, Shaw PA, Aplenc R, Barrett DM, Bunin $\mathrm{NJ}$, et al. Chimeric antigen receptor $\mathrm{T}$ cells for sustained remissions in leukemia. New England Journal of Medicine. 2014;371(16):1507-1517.

22. Louis CU, Savoldo B, Dotti G, Pule M, Yvon E, Myers GD, et al. Antitumor activity and long-term fate of chimeric antigen receptor-positive $\mathrm{T}$ cells in patients with neuroblastoma. Blood. 2011 Dec 1;118(23):6050-6056.

23. Pule MA, Savoldo B, Myers GD, Rossig C, Russell HV, Dotti $\mathrm{G}$, et al. Virus-specific T cells engineered to coexpress tumor- specific receptors: persistence and antitumor activity in individuals with neuroblastoma. Nature Medicine [Internet]. Springer Nature; 2008 Nov;14(11):1264-12670.

24. Bagley SJ, Desai AS, Linette GP, June CH, O'Rourke DM. CAR T-cell therapy for glioblastoma: recent clinical advances and future challenges. Neuro-oncology. 2018 Mar 2;20(11):1429-1438.

25. Germain RN, Stefanová I. The dynamics of $\mathrm{T}$ cell receptor signaling: complex orchestration and the key roles of tempo and cooperation. Annu Rev Immunol. 1999;17:467-522.

26. Buchbinder EI, Desai A. CTLA-4 and PD-1 pathways: similarities, differences, and implications of their inhibition. American journal of clinical oncology. 2016;39(1):98-106.

27. Chen L and Xue Han. Anti-PD-1/PD-L1 therapy of human cancer: past, present, and future. J Clin Invest. 2015;125(9): 3384-3391.

28. Topalian S L, Hodi F S . Safety, Activity, and Immune Correlates of Anti-PD-1 Antibody in Cancer. N Engl J Med. 2012 Jun 28;366(26):2443-2454.

29. LaFleur MW, Muroyama Y, Drake CG, Sharpe AH. Inhibitors of the PD-1 pathway in tumor therapy. The Journal of Immunology. 2018;200(2):375-383.

30. Ribas A, Wolchok JD. Cancer immunotherapy using checkpoint blockade. Science 2018;26(359):1350-1355.

31. Dashnamoorthy R, Chen B, Galera P, Chang H, Beheshti A, Ghosh S, Evens AM. The Immune Checkpoint Receptors PD-1, PD-L1, TIM-3 and LAG-3 in Lymphoma: Tumor Cell and Tumor Infiltrating Lymphocyte (TIL) Expression, Patient Prognostication, and Identification of Rational Therapeutic Targets. Blood 2017;130:2750.

32. Lichtenegger FS, Rothe M, Schnorfeil FM, Deiser K, Krupka C, Augsberger C, et al. Targeting LAG-3 and PD-1 to Enhance $\mathrm{T}$ Cell Activation by Antigen-Presenting Cells. Frontiers in Immunology [Internet]. Frontiers Media SA; 2018 Feb 27;9:385.

33. Chunqing G, Masoud HM, John RS, Devanand S, Paul BF, Xiang-Yang W. Therapeutic Cancer Vaccines: Past, Present and Future. Adv Cancer Res. 2013;119:421-475.

34. Olivera J. Finn. Vaccines for Cancer Prevention: A Practical and feasible approach to the Cancer Epidemic . Cancer Immunol Res. 2014 Aug;2(8):708-713.

35. Guo C, Manjili MH, Subjeck JR, Sarkar D, Fisher PB, Wang $\mathrm{XY}$. Therapeutic cancer vaccines: past, present, and future. InAdvances in cancer research 2013 Jan 1 Vol. 119, pp. 421-475.

36. Santos PM, Butterfield LH. Dendritic Cell-Based Cancer Vaccines. J Immunol 2018;200:443-449.

37. Palucka K, Banchereau J. Cancer immunotherapy via dendritic cells. Nat Rev Cancer. 2012;12(4):265-277.

38. Subramaniam D S. Novel Approaches to Cancer Immunotherapy. Discov Med 2016 Apr; 21(116):267-274

39. Thomas S, Prendergast GC. Cancer Vaccines: A Brief Overview. In Sunil Thomas (ed.), Vaccine Design: Methods and Protocols: Volume 1: Vaccines for Human Diseases, Methods in Molecular Biology, vol. 1403, April 2016.

40. Nemunaitis J . Sipuleucel-T in Metastatic Castrate-Resistant Prostate Cancer (mCRPC): Clinical Evidence of the Immunologic Mechanism of Action (MOA). Cancer- Immunotherapy: Harnessing Gene Therapy To Improve Anti-Tumor Effect. 2012 May; 20 (Supplement 1), S131.

41. Melero I, Gaudernack G, Gerritsen W, Huber C, Parmiani G, Scholl S, et al. Therapeutic vaccines for cancer: an overview of clinical trials. Nature reviews Clinical oncology. 2014 Sep;11(9):509-524. 
42. Raja J, Ludwig JM, Gettinger SN, Schalper KA, Kim HS. Oncolytic virus immunotherapy: future prospects for oncology. Journal for immunotherapy of cancer. 2018 Dec;6(1):140.

43. Becker $\mathrm{N}$ and Benhar I. Antibody-Based Immunotoxins for the Treatment of Cancer. Antibodies 2012;1:39-69.

44. Coulson A, Levy A, Gossell-Williams M. Monoclonal Antibodies in Cancer Therapy: Mechanisms, Successes and Limitations. West Indian Med J. 2014 Oct;63(6):650-654.
45. Ardolino M,Hsu J, Raulet D H. Cytokine treatment in cancer immunotherapy. Oncotarget. 2015 Aug 14;6(23): 1934619347.

46. Conlon KC, Miljkovic, Waldmann TA. Cytokines in the Treatment of Cancer. J Interferon Cytokine Res . 2019 Jan;39(1): 6-21. 